Bull. Austral. Math. Soc.

$62-07,62 \mathrm{P} 10$

VOL. 66 (2002) [527-527]

\title{
Risk factors associated with renal disease in diabetes mellitus
}

\author{
Yee Hung Choy
}

This thesis is concerned with the identification of risk factors associated with renal disease in diabetes mellitus. The goal of this research is to develop a mathematical model which can be used by clinicians as an aid in detecting risk factors for diabetic nephropathy. Hence it can be applied to patients with diabetes when they are at risk of developing renal disease, for there is evidence that renal disease is one of the most devastating of diabetic complications, being a major cause of death and an important predictor of cardiovascular disease.

The thesis starts with a complete review of the literature in order to give an overview of which risk factors should be of interest in the development of diabetic nephropathy. The research proper is divided into two parts. One is mainly theoretical, while the other is experimental. In the latter, longtitudinal data are utilized on 267 patients with diabetes to develop a statistical model. An extensive literature review is undertaken to compare, and possibly combine, those findings from the many relevant journal papers, which will enable one to identify those risk factors which effect the onset of diabetic nephropathy.

The risk factors obtained from both methods have been compared, and their similarities and discrepancies are discussed. A mathematical model has been formulated by applying logistic regression to the clinical data set. Recommendations for treatment, diagnosis of diabetic nephropathy, and suggestions for further research are also outlined.

\author{
Department of Applied Mathematics \\ Hong Kong Polytechnic University \\ Hung Hom \\ Kowloon \\ Hong Kong \\ e-mail: mayhchoy@inet.polyu.edu.hk
}

Received 20th August, 2002

Thesis submitted to the University of Technology, Sydney June 2001. Degree approved July 2002. Supervisors: Professor A.G. Shannon and Dr. P. Petocz.

Copyright Clearance Centre, Inc. Serial-fee code: 0004-9727/02 \$A2.00+0.00. 